vol. 26 - $n^{\circ} 2 \mid 2010$

Numéro ouvert

\title{
Migrants transnationaux et nouveaux réseaux criminels
}

Transnational Migrants and New Criminal Organizations

Emigrantes transnacionales y nuevas organizaciones criminales

\section{Alain Tarrius}

\section{(2) OpenEdition}

Journals

Édition électronique

URL : https://journals.openedition.org/remi/5151

DOI : 10.4000/remi.5151

ISSN : $1777-5418$

Éditeur

Université de Poitiers

Édition imprimée

Date de publication : 1 septembre 2010

Pagination : 155-167

ISBN : 978-2-911627-55-2

ISSN : 0765-0752

\section{Référence électronique}

Alain Tarrius, "Migrants transnationaux et nouveaux réseaux criminels », Revue européenne des

migrations internationales [En ligne], vol. 26 - n² | 2010, mis en ligne le 01 septembre 2013, consulté le 15 avril 2022. URL : http://journals.openedition.org/remi/5151; DOI : https://doi.org/10.4000/remi. 5151 


\title{
Note de recherche
}

\section{Migrants transnationaux et nouveaux réseaux criminels}

\author{
Alain TARRIUS*
}

ous avons mené une recherche ${ }^{1}$ sur la convergence entre des réseaux migra-
toires internationaux de vente de produits d'usages licites par les « transmigrants » et les réseaux criminels de trafics de psychotropes et de blanchiments. Cette première recherche nous a permis d'ouvrir un second volet envisageant l'usage qui se généralise de la vente de cocaïne par les travailleuses du sexe, en Espagne notamment. C'est ainsi que notre démarche nous a permis d'identifier des liens entre ces diverses pratiques délictuelles ou criminelles et l'actuelle recomposition des réseaux qui les développent.

\section{LA MONDIALISATION DES PAUVRES}

Deux types de réseaux et de circulations migratoires, qui œuvraient parallèlement, avec plus de contradictions et de conflits que d'affinités, sont en train de fusionner à l'occasion des derniers avatars de la crise financière du monde globalisé. D'une part, les vieux réseaux criminels, qui faisaient circuler les drogues, les armes et les femmes, suivant une logique territoriale de « pieuvre », c'est-à-dire une expansion omnidirectionnelle à partir d'une tête unique; en cas de risque de dévoilement, ils manifestaient cependant une forte capacité de fractionnement en micro unités, de repli sur des territoires sûrs, selon une logique de «famille localisée ». De telle sorte que l'observateur n'apercevait qu'une manifestation locale de cet ensemble criminel que l'on qualifie hâtivement de « mafieux » et que l'on dénomme différemment selon le lieu : mafia, n'draguetha, camorra, cosa nostra en Italie, « brise de mer » en Corse, « avant-garde géorgienne » en Catalogne, etc. D'autre part les nouvelles transmigrations, c'est-à-dire un type récent de migrations internationales, où des centaines de milliers de petits entrepreneurs nomades se déploient en réseaux de revente avantageuse de produits d'usages licites préférentiellement électroniques, non

* Professeur émérite, Université de Toulouse le Mirail ; altarrius@aol.com

1 Pour l'INHESJ (Institut National des Hautes Études en Sécurité et Justice) de fin 2007 à fin 2009

(A. Tarrius, S. Missaoui et O. Bernet). Je présente dans cet article mes propres enquêtes. Cette recherche poursuit celle que j'ai menée de 2003 à 2007 sur les transmigrants afghans pratiquant le commerce de produits « passed by Dubaï taxless » dans les Balkans (Tarrius, 2007). 
taxés, aux quatre coins de la planète, véritable force de travail transfrontalière commerciale de grandes firmes mondialisées, gênées dans leur expansion par les règles interétatiques du commerce officiel. Ces transmigrants effectuent en plusieurs mois des tournées, de chez eux à chez eux, et nulle part ne posent des problèmes urgents d'intégration aux sociétés qu'ils côtoient et traversent, travaillant brièvement aux champs et dans le bâtiment après avoir écoulé leurs marchandises. Leurs clientèles privilégiées sont les populations pauvres de nations pauvres ou riches : "poor to poor ", " pour et par les pauvres » est en effet un objectif commercial des grandes firmes dans leur déploiement. Un abaissement des prix de $40 \%$ sur les produits d'entrée de gamme de marques prestigieuse rend solvables, de par le monde, des centaines de millions d'individus. Là, les réseaux sont très lisibles, d'un bout à l'autre des distributions, et les marchandises commercialisées sont d'usage licite, mais évidemment échappent aux taxes usuelles.

\section{«INVISIBILITÉ » DES TRANSMIGRANTS?}

Les responsables étatiques, aussi bien que ceux des grandes ONG, s'abstiennent de reconnaître la nouvelle et importante forme des migrations internationales que représentent les transmigrants, dans un contexte où leurs activités ne sont pas criminelles. Par ailleurs, les analyses des réseaux criminels sont maintenues dans un flou propice à toutes les affabulations. Il apparaît relativement plus urgent et plus aisé de gérer les demandeurs d'asile et les sans papiers, que de reconnaître des populations en migration qui excèdent de toutes les frontières et ne demandent aucune intégration. Dans ces conditions, on comprend que le recouvrement actuel des réseaux de transmigrants par les réseaux criminels soit hors de portée des analyses des transformations des modes d'organisation de la criminalité ; c'est-à-dire échappent à la visibilisation.

Pour les ONG, ces migrants-là ne relèvent pas de leur action ${ }^{2}$. Ils leur apparaissent comme non nécessiteux et très extérieurs aux problématiques de l'assistance juridique. Pourtant, les situations sanitaires et affectives des transmigrants sont très proches des autres migrants internationaux de la misère.

Pour le moins, un pan de la réalité sociale et économique des migrations contemporaines est ignoré. C'est-à-dire la capacité d'analyse de toutes les circulations migratoires s'en trouve affectée : en effet, nous avons en tous lieux aperçu la porosité des diverses formes $^{3}$. Mais cette reconnaissance-là implique une vision autrement dynamique des mouvements planétaires des populations en migration. Le problème concomitant c'est la facilité d'expansion donnée ainsi à la nouvelle forme criminelle qui, pour se développer, associe de plus en plus intensément l'économie criminelle, la vieille « pieuvre », à la transmigration.

Les difficultés que rencontrent la plupart des paradigmes sociologiques et géogra-

2 Nous ferons une exception pour la CIMADE de Montpellier qui, au contact des Marocains transmigrants, a bien compris leur problématique et leurs besoins d'assistance.

3 C'est ainsi que, dans les Balkans les informations sur les migrants de la misère en Grèce sont abondantes et celles sur les nombreux transmigrants qui passent en Bulgarie sont inexistantes. Or, ces deux formes migratoires se recomposent en Macédoine. 
phiques à envisager le mouvement des populations dans la genèse de leurs sociabilités sont inhérentes à leur déploiement dans le cadre socio politique des États-nations. Le paradigme du « lieu » dans les sciences sociales et celui de l' « identité », conçue comme préséance locale du sédentaire sur le voyageur ou le nomade, ont réduit la mobilité à un état évanescent, transitoire, à un manque peu exploré, à une trajectoire mécanique d'un point à un autre. Pour notre part, on l'aura compris, nous réinvestissons les espaces-temps de la mobilité, du déplacement, des interactions sociales qui la caractérisent, qui font continuité, donnent pouvoir au migrant sur l'immobilité des dispositifs des États nations face aux désordres de la mondialisation. Nous avons ainsi proposé la notion de « territoire circulatoire " : les réseaux font sens pour nous par l'histoire de leur déploiement, par les statuts et rôles de leurs membres, par les interactions qui les solidarisent, mais aussi par la substance même des échanges, la marchandise et la réification de leurs mobilités en itinéraires transnationaux et donc trans-urbains.

\section{UNE NOUVELLE ET PUISSANTE FORME MIGRATOIRE CRIMINELLE}

L'évolution de la transmigration afghane, que nous étudions depuis six années, est caractéristique de ce mouvement. Cette forme migratoire internationale est née au début des années 2000 et fédère en plusieurs vagues des dizaines de milliers de migrants pendulaires, d'Afghanistan jusqu'aux Balkans et retour. Ces Afghans cumulent durant un, deux ou parfois trois semestres des petits emplois agricoles passagers avec le commerce de produits électroniques en provenance du Sud-est asiatique, et passés hors taxes par Dubaï ou Koweït, soi-disant destinations terminales. Ils se chargent de ces marchandises à Trabzon, port turc de la Mer Noire, ou à Samson, ou encore à Poti, en Géorgie, et les commercialisent dans les Balkans, principalement en Bulgarie. Ces commerces permettent d'écouler des marchandises à hauteur de six milliards de dollars environ. De grandes banques des Émirats, Bahreïn, Dubaï, et de Koweït-City, leur consentaient à partir de Trabzon, où ils reçoivent les marchandises acheminées par les aéroports côtiers, des lignes de crédit d'un montant moyen de $40 \%$ de leurs achats. Dès le mois de décembre 2008, ces banques refusèrent de renouveler ces crédits, l'opération étant illégale, puisque ces marchandises, hors taxes, ne pouvaient, suivant les engagements des États être réexportées dans de telles conditions des Émirats où elles arrivent en «destination terminale ». La Crise conduisait en effet à un durcissement des régulations bancaires dans le sens d'une adéquation des circulations monétaires aux règles politiques internationales de transfert des marchandises. Dès lors, des milieux turco-italiens troubles constituèrent des « petites sociétés » d'investissement, à partir de «placements » de 10000 à 20000 euros par cinquante à cent épargnants : inutile de dire qu'il s'agit de prête-noms pour de l'argent du blanchiment des dérivés du pavot à opium, morphine et héroïne, etc., que les Afghans doivent désormais cultiver au cours de leurs tournées, s'ils veulent obtenir le moindre crédit de ces « petites sociétés » d'investissement. Ainsi depuis une année, la transmigration afghane est soumise aux milieux criminels turco-italiens... pour aider des Turcs, des Arméniens et des Géorgiens, d'Abkhazie ou non, dans une paix que pourraient leur envier les responsables politiques, à implanter une industrie des psychotropes illicites et les firmes internationales de l'électronique du Sud-est asiatique à commercialiser leurs produits. 
Les dizaines de milliers de transmigrants afghans, qui jusqu'alors commettaient un délit fiscal, sont désormais les chevilles ouvrières de réseaux criminels en prêtant main-forte, pour des salaires dérisoires, à la tentative de culture du pavot somniferum dans ces régions et en blanchissant une partie de l'argent de ses transformations par l'achat des produits électroniques à livrer en Europe. Les mouvements pendulaires de leurs transmigrations sont désormais liés aux phases culturales du pavot requérant l'intervention de « main-d'œuvre spécialisée » et nombreuse. Ils provoquent la corruption de nombreux représentants des États, précisément dans les lieux de passage des produits et des personnes : douaniers, militaires, policiers, agents consulaires en premier lieu.

Les Afghans qui, après s'être délestés de leurs marchandises en Bulgarie, décident de rejoindre la migration « classique » vers la Grande-Bretagne par exemple, choisissent alors leur itinéraire à partir de la Macédoine : ils peuvent rejoindre la migration des pauvres, par la Grèce ou par Trieste. Le pécule qu'ils ont constitué à partir de leurs ventes en Bulgarie leur permet d'envisager le paiement des divers "péages » jusqu'à Londres, sans jamais se confondre avec les pauvres de la « jungle» de Sangatte. Soit ils rejoignent l'Italie du Sud, après un passage en Serbie, Monténégro et Albanie où ils augmentent ce pécule par des transports de psychotropes et surtout préparent ainsi leur accréditation auprès des milieux criminels sud-italiens. Un séjour à Naples, Brindisi ou Tarente à travailler pour des activités de blanchiment leur permet d'obtenir très rapidement des papiers, un complément de pécule et une introduction dans des milieux " industrieux » d'Angleterre, de Belgique ou des Pays-Bas ${ }^{4}$. Mais aussi, désormais, un rôle d'auxiliaires dans les réseaux criminels italiens.

\section{LES VOIES DE LA PROSTITUTION}

Les mêmes villes italiennes offrent une escale de deux ou trois semaines à de futures prostituées en route pour les « clubs » espagnols. Elles sont accompagnées par des Afghans, des Géorgiens ou des Ukrainiens et apprennent, pendant ce séjour, à maîtriser la confection des « doses de coke d'un quart d'heure » qu'elles proposeront ensuite à leurs clients procurant ainsi aux commanditaires espagnols des revenus plus substantiels que ceux de la prostitution ou du « deal » seul. L'histoire de trois d'entre elles est exemplaire :

\section{Sardinella l'Albanaise}

Lorsque je rencontrai ${ }^{5}$ Sardinella elle avait vingt-sept ans. Elle travaillait dans un « club d'abattage ${ }^{6}$ » proche d'Alméria depuis environ vingt-six mois en qualité de " barmaid », en réalité à la passe. En attente de sa régularisation définitive, elle avait projeté, une fois cette démarche accomplie, de retourner à la ferme proche de Shkodra, en Albanie, où elle était née et que ses parents exploitaient toujours; elle possédait un frère de deux ans son aîné. Sa « carrière » en Espagne avait débuté à La Junquera, six années

4 Tarrius et Bernet, 2010.

5 Entretiens avec A. Tarrius, d'une durée de vingt-trois heures.

6 Il s'agit d'un club pour ouvriers agricoles étrangers, surtout marocains dans ce cas. 
auparavant, sur la frontière franco-espagnole, s'était poursuivie dans un club d'abattage - pour ouvriers agricoles étrangers - près de Valencia, puis dans un petit club sur une plage d'Alicante et enfin de nouveau dans un club d'abattage, près d'Almería, soit au total sept années. Tout cela après un passage de deux années à Tarente, dans le Sud italien. Sardinella s'exprimait dans un excellent italien.

«Après 1989, mes parents avaient renoué avec la tradition familiale catholique. Ils me firent donc baptiser cérémonieusement par un prêtre italien. À mon prénom chrétien on ajouta celui de "Sardinella", un peu par dérision... celui-là même qui allait me rester ; j'étais grande, très maigre avec une petite tête et des yeux ronds : "ni bonne à griller, trop sèche, ni bonne à saler, trop longue : ni sardine ni anchois,

c'est une sardinelle" avait dit le prêtre; et je suis restée ainsi. Avec mon surnom, que j'aimais bien...

C'est à quinze ans que l'envie me vint de partir pour l'Italie. Ici, dans le nord de l'Albanie nous sommes très liés à la région de Tarente et Brindisi; les religieux et les bonnes sœurs qui viennent chez nous attirent là-bas des travailleurs saisonniers ou définitifs. Pas les voyous musulmans qui vont dans les Abruzzes, de bons ouvriers agricoles et des pêcheurs aussi catholiques que les Italiens. Alors, je suis passée par les religieuses. C'était la voie. Deux années de messes et de vêpres. Et puis le grand jour : le noviciat à Tarente.

On s'est embarqués à Durrës, pour Brindisi, trois religieuses italiennes, un curé et moi, en robe blanche de novice.

Arrivée à Tarente j'ai été en noviciat une année dans un grand appartement bourgeois aménagé en couvent; une vie tranquille mais un peu triste. L'Italie était dehors... si tu vois ce que je veux dire. J'avais dix-huit ans, et on m'a donné, lors d'une petite fête, des papiers de résidente. Mon bonheur était fait, celui de ma famille aussi.

Mais le soir même je m'enfuyais dans les rues de Tarente, précipitant dans le malheur tous ceux qui, au même moment, me fêtaient.

Dans cette ville, lorsque tu es en rupture avec les religieux, les bourgeois et ta famille, tu n'as pas de choix, il faut habiter sur l'île, entre les deux bords de la lagune et en face du Golfe. Il y a là de vieux immeubles de trois ou quatre siècles, complètement pourris et peuplés de zombies qui sortent la nuit pour la came. Vers le Golfe des remparts d'environ dix mètres et vers la lagune le port de pêche et la grande criée aux poissons.

Le quai est large et l'eau affleure. Il y a un café-tabac pour des fainéants ivrognes, de ceux que la mafia n'a même pas voulu employer pour nettoyer la criée. Et des types alignés contre les vieilles façades, les mains dans les poches, bien écartées, de huit heures du matin jusqu'à la nuit. Ensuite, c'était ma vie qui commençait...

C'est là que j'ai rencontré Emilio, un faux dur de vingt-deux ans qui travaillait de temps à autre avec un pêcheur qui lui fourguait un peu de mauvaise coke et des poissons invendables pour le payer. Autant te dire qu'Emilio, il a tout de suite été pour moi. Il était petit et gros, alors, tu vois le couple. Mais depuis trois ans qu'il rôdait dans le coin, il s'était fait une niche dans un vieil immeuble qui ne prenait pas la flotte. J'ai trouvé un petit boulot à la criée et nous avons vécu comme des oiseaux au nid, ou comme des rats au fond du trou, c'est selon qu'on voit la vie comme deux

tourtereaux adolescents ou comme deux adultes ratés, là il s'agissait bien de

ratage. Pas de dérapage, tu vois, un virage raté au-dessus d'une falaise, et depuis sept ans je n'en finis pas de chuter vers le fond de je ne sais quel précipice. Emilio et son pêcheur m'expliquèrent qu'il serait bon pour tous que je travaille trois ou quatre heures, jusqu'à minuit, en me vendant dans la grande barque de pêche qui possédait 
une minuscule cabine "tu n'as pas besoin de t'allonger, les clients ils préféreront que tu fasses tout ça à genoux" me dit le pêcheur en riant. Comme ils m'aimaient bien, ils m'expliquèrent encore que je ferai la passe avec de la coke : à moi de la doser pour que le cave s'énerve sans pouvoir passer à l'acte; et surtout qu'il ne s'endorme pas. Et pas d'overdose sinon il faudrait les jeter dans la lagune - ce qui n'est jamais arrivé -. C'était une exigence des mafieux qui voulaient un jour me récupérer avec ce "savoir-faire" ; disons qu'une fois sur deux j'évitais les envies de mes clients ; à ceux qui revenaient et qui demandaient leurs fantasmes (...), je disais

que j'avais le Sida, mais qu'ils ne risquaient rien car j'allais me laver à l'eau de mer qui nous entourait, et qui est une des plus polluées d'Italie; la coke leur suffisait alors. Et je ne les revoyais pas. Nous avons vécu quelques mois ainsi,

Emilio avec des surplus de coke et mes sous et le pêcheur avec son commerce de dope; et puis il a fallu travailler plus pour des policiers qui nous avaient repérés. Et tout se termina par la grande transaction finale : les mafieux envoyèrent le rafiot du pêcheur par le fond, à l'aide d'un bidon d'essence et triplèrent le volume de sa tête et de celle d'Emilio, après leur avoir arraché un cil ; non seulement mes associés ne payaient rien, mais ils traitaient ouvertement les mafiosi d' "aveugles". Ils s'en tirèrent borgnes, somme toute c'était généreux. On m'embarqua dans une grande vedette:

"La coke et le sexe, ça marche fort en Espagne, et puis t'es tellement moche que les vicieux aiment ton genre ; alors demain matin, direction Barcelone". Avant de partir, ils m'envoyèrent faire quelques courses, pour les trois repas à venir. J'ai acheté pour une misère cinq kilos de... sardinelles. L'un d'eux me dit "bien! du poiscaille, ça nous donnera des forces pour te faire la fête" ; ce qui devait arriver arriva, dès le premier repas l'insupportable odeur de la sardinelle frite les fit vomir ; le curé aurait pu dire, lors du baptême, que je ne pouvais pas davantage être frite. Les pêcheurs me mangent crue. Je devais bien ça à Emilio. Bref, ils me considérèrent comme une vraie catastrophe; et le dégoût que je leur inspirais me réconforta d'un voyage passé sur le pont dans les embruns "pour pas, en plus, que tu pues trop

à l'arrivée, et nous avec'. Il y avait là un Italien, le pilote, un Géorgien, et deux Afghans.

Le débarquement s'effectua au port de La Escala. Ils me dissimulèrent un peu, probablement qu'ils avaient peur d'être déconsidérés, surtout parce qu'ils transportaient autre chose : de la belle neige de l'Etna, comme on dit en Italie. Moi j'étais le pourboire. Je ne les ai pas enrichis, et heureusement que j'avais le permis de circuler italien, sinon personne ne m'aurait prise, y compris dans le club le plus lamentable...

Des transactions suivirent mon arrivée dans un "club" de la Junquera qui comprenait une vaste cour pour le stationnement de camions au passage de la frontière, un restaurant, une boutique d'alcools et de conserves et, dans un bâtiment en rez-dechaussée, le bar et les chambres du bordel. Il y a six chambres; les filles sont louées au quart d'heure.

Au début, en attendant d'avoir des papiers espagnols en règle, je faisais les nettoyages des chambres et du bar dans la journée. La nuit, j'allais dans un bosquet voisin "travailler pour les flics" : un fourgon se plaçait là tous les soirs de neuf heures à minuit pour la "sécurité" qu'ils disaient ; en fait, c'étaient mes proxénètes flics. Je leur reversais la recette, et ils n'oubliaient pas la passe... Après minuit j'orientais les clients qui voulaient la passe + la coke vers mon hôtel à la Junquera ; des Africaines y louaient aussi des chambres. Chaque soir, un gars venait de Perpignan avec les doses toutes prêtes : on le payait cash au prix fort. On faisait au maxi quatre caves chacune, jusque vers deux heures du matin. C'étaient mes seuls 
revenus ; au total 800 euros par mois, à cause du prix de la coke; mais les clients de l'hôtel en voulaient. Lorsque j'ai eu mes premiers papiers provisoires, à la fin de l'année suivante, tout a changé. On m'a officiellement embauchée au club, comme "serveuse" ; à la passe, j'étais d'une rentabilité très moyenne, une dizaine de clients entre six heures de l'après-midi et environ deux heures du matin. Ils me disaient sans arrêt que le plancher devait être de deux à l'heure. Mes clients : peu de camionneurs - je n'étais pas leur idéal de femme - et beaucoup de Français un peu solitaires et - ma malédiction - un peu bizarres ; le club commençait à prendre une tournure un peu sinistre. C'est alors, comme "dernière chance" qu'on m'a mise aux enchères; les principaux clients de Perpignan ont été avertis et un vendredi, entre dix-sept et dix-huit heures, une dizaine était là, rideau tiré. On m'a regardée sous toutes les coutures et le patron a proposé " 5000 euros; à partir de 5 mises rapport $8 \%$ ". Les clients se tapaient sur les cuisses en riant et l'un d'eux dit : "rien à moins de $15 \%$; tu nous refiles la grande Duduche" ; je hurlai "je suis Sardinella !! Gros porc de Français" après un instant de silence, ils explosèrent de rire. "Et en plus elle est hystérique" s'esclaffa un petit gros dont on me dit par la suite qu'il était juriste. Les bourgeois français qui se lâchent n'ont rien à envier aux Italiens paumés du port de pêche de Tarente. Le patron arrêta les enchères et leur offrit un repas. Puis il vint me voir, dans une chambre; là je reçus ma première sérieuse correction, c'est-à-dire avec la boucle de la ceinture: "fais ta valise, demain tu descends au Sud". À l'hôtel, ce soir-là le veilleur vint me dire "je ne peux plus te garder... puis avec une douceur que je n'avais pas entendue depuis longtemps... Sardinella, je te regarde et je t'aime beaucoup ; alors, crois-moi, tu n'es pas faite pour faire la pute, enfuis-toi en France; ils vont te mettre dans un bordel d'abattage pour les ouvriers

agricoles arabes, près d'Alicante”. Je me mis à pleurer : c'était le seul homme depuis longtemps qui me parlait avec douceur. Je ne voulais pas l'embêter, alors sans rien dire je suis allée faire ma valise, et j'ai dormi chez une Africaine qui m'a longtemps serrée contre elle, après m'avoir montré des cicatrices de cigarettes sur son dos. "Tu vois me dit-elle, moi, c'est comme ça qu'ils ont mis la haine dans mes tripes. Si tu peux revenir chez toi, va-t-en; pour moi c'est impossible et maintenant je sais que je mourrai dans la misère et la maladie".

Somme toute, cette maison d'abattage était bien plus confortable que mes "hébergements" précédents : douze heures de travail par jour, dix minutes par client, vingt pour nous, y compris le nettoyage du lavabo et du drap. Ils n'avaient pas le choix, et donc (...) sans autre fantasme que l'acte normal. On m'avait dit que j'aurais affaire à des Arabes, et que ce serait terrible. En fait, ils étaient plus propres, sur eux et dans leur tête que les tordus italiens et français. Dans ces conditions je faisais bien mon travail et au bout de quatre mois je fus "cédée" à un petit club proche d'Alicante, sur une plage. Les employés espagnols d'une grande boîte européenne fournissaient la clientèle: trois heures de bar et de nettoyage, six heures de travail en chambre à l'intérieur, et les deux dernières heures dehors, avec la coke, qui était livrée chaque jour par un Hollandais, en doses. Ensuite je dormais dans une des six chambres où je travaillais. Ça aurait pu être bien, si j'avais eu la clientèle des ouvriers agricoles arabes, mais le défilé des neuneus avait recommencé-ils m'avaient vite repérée -, avec leurs jérémiades et leurs demandes de tordus.

L'inévitable se produisit ; le patron me dit "désolé Sardinella, je t'aime beaucoup, mais bientôt nous aurons la réputation de l'annexe de l'hôpital psychiatrique ; et ça c'est pas bon pour le commerce". Il m'a refilée à un bordel d'abattage près d'Almería ; c'est là que tu m'as trouvée; c'est la dernière étape avant l'Afrique, aussi il est temps que je rentre à Shkodra. J'y pense depuis au moins deux ans. Il ne me reste plus que quatre mois pour avoir les papiers définitifs (...)». 


\section{Chrisna la Géorgienne}

De la région de Jarie, en Géorgie, sur la Mer Noire, limitrophe de la Turquie, Chrisna (diminutif de Christina) avait vingt-huit ans lorsque je la rencontrai dans un « club abattoir » entre Malaga et Almería. Elle était arrivée en Espagne sept ans plus tôt. Ses grands-parents étaient des émigrés arméniens, des catholiques arméniens. Son père avait créé une petite entreprise de démontage de coques de bateaux, bois et acier, qui comptait trois équipes de cinq ouvriers. Ils travaillaient près du port de Poti, et bénéficiaient du fort développement des échanges maritimes dans ce secteur. Chrisna s'exprimait dans un espagnol correct. Son histoire est, si l'on peut dire, limpide.

« $\grave{A}$ dix-huit ans, après une formation professionnelle et des diplômes, mon père m'embaucha pour tenir la comptabilité, prendre les commandes, etc. Au port, je connaissais les marins de passage; nous allions gentiment prendre un verre avec certains. J'ai alors connu un Russe qui était accompagnateur d'un riche entrepreneur du Golfe. Il était basé à Trabzon à environ deux cents kilomètres de chez nous. Mon père voyait d'un très mauvais oeil cette liaison. Alors, c'est classique, je suis partie avec le Russe. Trabzon n'était pas loin mais l'histoire de cette frontière,

les Arméniens, puis le socialisme, donnait l'impression d'une grande distance. À

Trabzon, nous étions pauvres, étrangers, et surtout non musulmans. Nous avons donc loué un petit deux pièces pour trente euros par mois au quartier du port. Mais mon ami partait souvent pour trois ou quatre semaines et de nombreux voyous géorgiens habitaient dans le quartier. L'un d'entre eux, la cinquantaine, qui ne manifestait aucune violence, me proposait souvent d'aller avec lui en Espagne où j'aurais un travail et de la protection.

Un jour, je l'ai sollicité ; mon copain partait pour quatre semaines. Je ne pouvais plus supporter ces périodes de solitude dans ce quartier pourri.

J'avais vingt-et-un ans, le Géorgien cinquante-quatre. Nous avons pris le bus jusqu'à Istanbul. Nous devions prendre l'avion à l'aéroport international pour Rome, puis, après un changement, pour Gérona, en Espagne. Là-bas nous étions attendus. À l'aéroport, les policiers emmenèrent mon compagnon, ils me dirent de ne pas me faire de souci, et de continuer mon voyage, son arrestation pouvant durer quelques semaines. Je leur avais dit qu'un travail m'attendait là-bas. Mais, avant l'embarquement, les policiers l'avaient relâché. Pour deux mille euros passés de main à la main.

Arrivés à Rome mon accompagnateur me dit que nous devions aller à Naples une semaine. J'ai connu pendant huit jours une consommation folle de cocaïne et de krach. Il fallait se préparer à l'Espagne me disait-il.

[...] Arrivée à Gérone, un Géorgien et un Afghan m'attendaient ; ils me conduisirent dans des clubs à la frontière qui refusèrent de m'employer après avoir écouté mon histoire. Un des patrons me dit "Marie-toi avec un vieux qui a de l'argent".

L'Afghan, qui était là sur ordre d'une sorte de "garde" de plusieurs clubs, abandonna là l'accompagnement. Le Géorgien était furieux. Nous sommes partis à quatre heures du matin pour Malaga. En route il m'a fait changer de vêtements,

style "light", m'a obligée à mettre du rouge à lèvres violet, et m'a collé un faux tatouage sur le bras. Ça tournait à la communiante dévergondée. Je lui ai dit : "ne

te fatigue pas, je sais qu'il s'agit de prostitution; je vais bien me vendre". Il me conduisit donc dans ce club près de Malaga. Il s'agissait d'un hôtel borgne pour

les ouvriers agricoles marocains (...) Ils gagnaient six cents euros par mois et la passe de dix minutes leur coûtait quinze euros. Je travaillais six heures au club, puis 
dehors quatre heures près des jardins, avec la dose de coke que me remettait un jeune Gitan, qui la tenait de Hollandais. Ils m'avaient loué un studio dans le vieux quartier près des jardins.

Cela a duré six ans, jusqu'il y a quelques mois. Maintenant je travaille dix heures, de quatre heures de l'après-midi, à trois heures du matin, avec une heure pour le repas. Nous sommes quatorze filles. Nous nous entendons bien. Une jeune vient d'arriver d'Azerbaïdjan. Elle avait commencé le métier en Turquie.

Moi, je vais partir bientôt. J'arrête : il n'y a aucun espoir dans ce boulot, sinon finir avec un de ces ouvriers marocains et crever de misère et de maladie. En sept ans,

j'en ai vu partir avec le Sida. Trois mois à l'hôpital, où les hommes nous sifflent quand on va les voir ; et après, où vont-elles? Pas vers leurs projets d'évasion du bordel.

Ici ils m'ont donné de l'héroïne: "Tiens, ça vient de chez toi, c'est de l'Afghane", m'a dit un jour un Italien qui confondait Est, - hérö̈ne, - Afghans versus Ouest coke-Amériques, bref, l'OTAN versus les Talibans. Les filles de l'Est nous sommes au 'régime héro', en prévision de notre retour... ».

\section{Farah la Tunisienne}

Originaire d'une ville sur la frontière tuniso-algérienne, et membre d'une fratrie de sept, dont un garçon et deux sœurs aînées, Farah est allée à l'université de Tunis à dix-sept ans. Ses parents, livreurs de légumes, étant dans l'incapacité de pourvoir à ses dépenses, elle travailla dans les vieux souks, à la vente «d'antiquités ». Lorsque je la rencontrai, elle travaillait, comme pensionnaire, dans un club confortable, près de Murcia, où elle était arrivée directement quatre ans auparavant. Elle avait alors vingt-deux ans.

«Tous les jours, matin et soir, je passais par des ruelles, entre les souks et une vieille porte de Tunis. C'était là que s'entassaient les prostituées, dans des rez-dechaussée sordides. Je n'ai jamais envisagé de me prostituer ainsi, par contre j'ai découvert des visages de femmes, et, à force de les saluer je leur ai parlé. Elles m'ont recommandé d'aller à Sidi Bou Saïd et sur l'avenue Bourguiba et de me faire draguer par des touristes propres et fortunés. Ici, dans ces rues, il y avait surtout des pauvres, et beaucoup de malades.

Au début, avec les étrangers, on ne savait où aller : impossible dans leur hôtel, impossible à la cité U. Puis j'ai pu louer quelque chose avec trois autres étudiantes. Moi, je disposais de la chambre de quinze à dix-huit heures. Je faisais deux ou trois étrangers, bientôt les mêmes, qui aimaient une jeune et belle Tunisienne.

$C$ 'est allé très vite. À dix-huit ans, une relation avec un officier des douanes m'a permis d'avoir un passeport puis un billet d'avion pour l'Espagne J'aurais pu, à l'époque, partir directement pour le Golfe, mais je ne les avais encore jamais rencontrés, et je me voyais en femme cloîtrée et battue. C'est des Espagnols qui ouvrent une boîte tous les étés qui m'avaient organisé le déplacement. Il n'y avait rien de compliqué : ils savaient que je voulais travailler, ils savaient qu'à Tunis des Espagnols m'avaient bien appréciée. Alors, c'est un joli club qu'ils avaient démarché, au sud de Murcia. La proposition était, sous réserve d'un mois de travail "pour voir" : cinq mille euros à l'Espagnol de Tunis, quatre mille pour moi à mon arrivée, puis sept mille mensuels, avec une retenue de deux mille pour la location d'une chambre, pour le médecin et pour le confort corporel. Le gérant, en plus, me proposait de mettre de côté 1500 euros mensuels, avec $5 \%$ d'intérêt. Évidemment j'ai été d'accord. La boîte fait bien 
les choses: tous les mois, on reçoit le relevé de ses gains ; pas les miens, ceux du patron. Pour moi, je tourne autour de 15000 euros, soit 180000 par an : une petite entreprise; et nous sommes trente-sept filles!

Mon travail consiste surtout à passer des soirées et des nuits avec des types du Golfe. Dubaï d'abord. Parfois je "suis de club". Dix heures; la passe est facturée par demi-heure cent euros. La coke se consomme chez les émirates. Ils m'en fournissent pour quand je ne sors pas.

En fait, je suis bien avec tout le monde et mes clients aiment bien parler avec moi. J'ai appris depuis longtemps à "baisser la garde des mots". Tu sais ce que veut dire baisser la garde? Un boxeur qui ne veut plus continuer un combat qui tourne à la boucherie; un guerrier qui préfère s'exposer que continuer davantage un combat sans raison; c'est se rendre dépendant de son adversaire sans perdre sa dignité. Pour les mots, c'est pareil, tellement de gens les utilisent comme des armes, couteaux ou boucliers, les combinent parfois avec des silences non moins meurtriers. Moi, quand je les vois dans cet état, je baisse ma garde des mots : je m'excuse, même si je n'ai pas tort, je suis sourde à une provocation. Et ils se calment; seulement un moment car ces états-là signifient beaucoup de souffrances, et depuis longtemps. Chez nous ils prennent cela pour de la soumission. Mais ils sentent bien qu'il y a autre chose, de la dignité, quoi. Je vais partir à Dubaï au mois de septembre. Plus que trois semaines à passer ici. Le gérant essaiera de tirer le maximum. Je crois qu'il a négocié autour de dix mille

euros.

Nous ferons les formalités pour le mariage ici, avant mon départ. Celui avec qui je vais vivre paie mon accompagnement par deux Espagnols, jusqu'à ce que tout soit en règle à Dubaï.

Je ne garderai pas un grand souvenir de mon séjour ici, sinon l'année dernière, en été, lorsque je suis allée sur un bateau qui faisait les clients des ports de Tarragone, de Sète, de Cannes. La prochaine étape, dans quelques années, ce sera un retour chez moi, avec beaucoup de bien-être ».

Somme toute on nous expose là des structures de contes enfantins; de la famille à la famille en passant par un ailleurs qui renverse les valeurs : jusqu'à leur retour, consenti cette fois. Les itinéraires ramènent chez soi après des étapes sur une sorte de territoire circulatoire des travailleuses du sexe.

\section{VERS DE NOUVELLES FORMES}

Ce que l'on peut apercevoir des morphologies des nouveaux réseaux criminels, sous réserve de recherches plus précises, c'est d'une part leur fragmentation en itinéraires multiples et d'autre part leur ethnicisation et leur « démocratisation », dans le sens d'une plus grande accessibilité des réseaux criminels par les populations locales.

\section{Fragmentation en itinéraires multiples non conflictuels}

De l'Europe de l'Est jusqu'à la France et l'Espagne on peut distinguer au moins six réseaux : celui qui, partant des ex-républiques soviétiques d'Asie, passe par la Pologne et l'Allemagne ; celui qui, partant du Caucase, passe par la Géorgie et l'Ukraine, puis l'Autriche et la Suisse ; celui qui, partant de Géorgie et d'Afghanistan, passe par la 
Turquie, l'Italie du Sud et l'Espagne, sur lequel a porté notre recherche ; celui qui, partant d'Asie du Sud-est, passe par le Golfe, le Liban, la Grèce, l'Italie du Nord et la France, « animé » par des Chinois ; celui qui, partant d'Afghanistan, passe par la Turquie, la Grèce, le sud de l'Italie, la France et l'Espagne ; celui qui, partant du Liban, traverse les pays sud-méditerranéens jusqu'en Espagne. Nous n'envisageons pas là les réseaux remontants (i.e. Nigéria, Maroc, France, Grande-Bretagne ; ou encore Somalie, Libye, Italie du Sud) ni transatlantiques (Colombie, Brésil, Europe et Afrique, etc.). Il ne s'agit là que des réseaux véhiculant cocaïne, crack, héroïne, morphine et brown sugar et, selon nos observations accompagnant à de nombreuses occasions des femmes pour des activités prostitutionnelles. Évidemment, cette multiplicité vit sans arrêt des chevauchements et irrigue souvent les mêmes régions. L'offre est ainsi diversifiée, tant en qualité qu'en prix, et elle couvre au mieux les territoires. On est passé d'un commerce de petits épiciers rivaux à une offre de type grands magasins multimarques. Quant aux classiques formations criminelles, italiennes par exemple, elles occupent désormais des points de passage clefs, confortant, sans perdre leur vocation locale, leur nouveau rôle dans les déploiements criminels mondiaux. Il n'y a plus de grands conflits entre réseaux. Cette évolution a été concomitante chez les transmigrants, comme dans les réseaux criminels. Il serait opportun d'identifier et de décrire des lieux de rencontre des réseaux et de comprendre les règles qui président aux diverses négociations et transactions.

\section{Ethnicisation}

Depuis une vingtaine d'années ${ }^{7}$ nous avons constaté que les transmigrants fonctionnent en réseaux ethniques et ont la particularité de développer un code d'honneur, un métalangage commercial, débarrassé des références religieuses ou idéologiques des uns et des autres, qui non seulement évite les conflits, mais de plus met en synergie la diversité pour l'obtention et la distribution d'un produit. Cette caractéristique qualifie aujourd'hui les nouveaux réseaux criminels. Chaque réseau possède la réputation de ses membres, Géorgiens obstinés, Chinois habiles commercialement, etc., mais sans que cela dépasse les représentations populaires usuelles et de façon même à valoriser les uns et les autres. Tous ces réseaux « ethniques » sont solidaires face à la diversité des politiques des États concernant les étrangers et face à la nécessité de partager un immense marché. Chez les prostituées étrangères qui travaillent en Espagne nous avons noté une concordance des étapes et de leurs durées selon les origines : si les recrutements s'effectuent souvent au cas par cas, une fois le séjour espagnol entamé, des milieux homogènes se créent autour de l'origine et fonctionnent comme autant de groupes ethniques.

\section{Démocratisation}

Ce ne sont plus des individus spécialisés dans le travail de rabatteurs qui recrutent clients et complices, mais des personnes attirées par les consommations ou l'opportunité de gains faciles, qui ouvrent, lorsque l'opportunité se présente, un petit marché de psychotropes en s'adressant au réseau le plus proche ou le plus visible. De même pour la prostitution, c'est l'occasion qui provoque l'intervention d'un loueur d'appartements ou

7 Tarrius, 1991, 1995. 
d'un tenancier de « boîte » dans l'espace méditerranéen et balkanique, auprès d'un gérant de club espagnol. Chacun de ces nouveaux partenaires peut fournir une entrée dans les différents réseaux criminels où participent de plus en plus d'individus aux profils sociaux « irréprochables » ou banals.

De plus en plus les «carrières » de prostitution, en Espagne, sont enclavées, durant quatre à sept ans, dans une histoire de vie familiale et sociale qui banalise ce " moment" en faisant continuité entre l'avant et l'après prostitution ${ }^{8}$. Cela caractérise les femmes originaires des Balkans. Pour nombre d'entre elles, il semblerait cependant qu'après des années de pratique de « la passe avec la dose de coke », l'usage de l'héroïne, très accessible dans ces régions d'origine, se chronicise. Le retour, si fréquent, des prostituées de l'Est vers leur milieu social d'origine est marqué pour plus de $50 \%$ d'entre elles par l'importation du VIH ou du VHC et de l'addiction à l'héroïne. Et demain?

Comme toujours les mouvements sociaux ont un temps d'avance sur l'organisation des pouvoirs et sur leur compréhension des changements. Mais cette fois il s'agit d'un changement de paradigme : l'association entre firmes transnationales, migrants internationaux, milieux criminels et diverses polices se généralise et se popularise à l'échelle de la planète dans un contexte de globalisation, de mondialisation.

\section{Références bibliographiques}

BATTEGAY Alain (2005) Dubaï : économie marchande et carrefour migratoire. Étude de mise en dispositif, in Hana Jaber et France Métral Éds., Mondes en mouvement, migrants et migrations au Moyen-Orient au tournant du XXIe siècle, Beyrouth, IFPO.

BAYART Jean-François (2004) Le gouvernement du monde. Une critique politique de la globalisation, Paris, Fayard, 448 p.

BAZIN Marc, KANÇAL Salgur, THOBIE Jacques et TEKELIOGLU Yavus (2000) Méditerranée et Mer Noire entre mondialisation et régionalisation, Paris, L'Harmattan, $520 \mathrm{p}$.

BORDES-BENAYOUN Chantal et SCHNAPPER Dominique (2006) Diasporas et nations, Paris, Odile Jacob, $222 \mathrm{p}$.

BOUBEKER Ahmed (2001) Des mondes de l'ethnicité, Thèse de sociologie, EHESS, Paris.

BOUGAREL Xavier et CLAYER Nicolas (Dir.) (2001) Le nouvel islam balkanique. Les musulmans acteurs du post communisme, 1990-2000, Paris, Maisonneuve et Larose, 500 p.

BREDELOUP Sylvie (1993) Les migrants du fleuve Sénégal : à quand la diams'pora ?, Revue Européenne des Migrations Internationales, 9 (3), pp. 205-232.

BRUNEAU Michel (2004) Diasporas et espaces transnationaux, Paris, Economica, 249 p.

CHARBIT Yves, HILY Marie-Antoinette et POINARD Michel (1997) Le va-et-vient identitaire. Migrants portugais et villages d'origine, Paris, INED-PUF, $144 \mathrm{p}$.

DERENS Jean-Arnault (2008) Balkans : la mosaïque brisée. Frontières, territoires et identités, Paris, Cygne, 208 p.

DIMINESCU Dana (Dir.) (2004) Visibles mais peu nombreux. Les circulations migratoires roumaines, Paris, MSH, $340 \mathrm{p}$.

8 Mes enquêtes sur les clubs espagnols de prostitution en 2008 et 2009 dénombrent $70 \%$ de retours dans les familles balkaniques après quatre à sept ans de prostitution. J'ai rencontré plus de 160 prostituées étrangères (Voir Tarrius et Bernet, 2010). 
ESCOFFIER Claire (2006) Migrations transsahariennes et communautés d'itinérance, Thèse de sociologie, Toulouse Le Mirail.

GOKALP Anton (2000) Migrations transnationales et nouvelles diasporas, in Académie universelle des cultures, Migrations et errances, Paris, Grasset.

HALBWACHS Maurice (1942) La topographie légendaire des Évangiles en Terre Sainte, Paris, PUF.

HANNERZ Ulf (1983) Explorer la ville, Paris, Les Éditions de Minuit, 432 p.

MA MUNG Emmanuel (2005) La diaspora chinoise et la création d'entreprises : réseaux migratoires et réseaux économiques en Europe du Sud, in Louis Muller et Stéphane de Tapia Éds., Un dynamisme venu d'ailleurs : la création d'entreprises par les immigrés, Paris, L'Harmattan, pp. 91-108.

MEDAM Alain (1993) Diaspora/diasporas. Archétype et typologie, Revue Européenne des Migrations Internationales, 9 (1), pp. 59-66.

MISSAOUI Lamia (1995) Généralisation du commerce transfrontalier : petit ici, notable là-bas, Revue Européenne des Migrations Internationales, 11 (1), pp. 53-75.

MISSAOUI Lamia (2003) Les étrangers de l'intérieur : filières, trafics et xénophobie, Paris, Payot, $274 \mathrm{p}$.

PARK Robert Ezra (1955) The collected papers of Robert Ezra Park, Glencoe, The Free press, $403 \mathrm{p}$.

PERALDI Michel (Dir.) (2002) La fin des norias? Réseaux migrants dans les économies marchandes en Méditerranée, Paris, Maisonneuve \& Larose, 493 p.

PEROUSE Jean-François (2002) Migrations, circulations et mobilités internationales à Istanbul, Les dossiers de l'IFEA, 13, pp. 10-29.

POPOVIC Anton (1994) Cultures musulmanes Balkaniques, Istanbul, Isis, 283 p.

SIMMEL Georg (1981) Sociologie et épistémologie, Paris, PUF.

SIMON Gildas (1995) Géodynamique des migrations internationales dans le monde, Paris, PUF, $429 \mathrm{p}$.

SOULTANOVA Ralitza (2005) Les migrations multiples de la population bulgare. Actes du colloque, La France et les migrants dans les Balkans : un état des lieux, Courrier des Balkans, http://balkans.courriers.info/article5504.html.

TARRIUS Alain (1989) Anthropologie du mouvement, Paris, Paradigme, 192 p.

TARRIUS Alain (1992) Les Fourmis d'Europe. Migrants riches, migrants pauvres et nouvelles villes internationales, Paris, L'Harmattan, $210 \mathrm{p}$.

TARRIUS Alain (1995) Arabes de France dans l'économie mondiale souterraine, La Tour-d'Aigues, L'Aube, $220 \mathrm{p}$.

TARRIUS Alain (2001) Les nouveaux cosmopolitismes. Mobilités, identités, territoires, La Tourd'Aigues, L'Aube, 266 p.

TARRIUS Alain (2002) La mondialisation par le bas. Les nouveaux nomades de l'économie souterraine, Paris, Balland, $220 \mathrm{p}$.

TARRIUS Alain (2007) La remontée des Sud : Afghans et Marocains en Europe méridionale, La Tour-d'Aigues, L'Aube, $201 \mathrm{p}$.

TARRIUS Alain et Olivier BERNET (2010) Migrations internationales et nouveaux réseaux criminels, Canet, Trabucaire, $160 \mathrm{p}$.

TARRIUS Alain et Olivier BERNET (à paraître) Migrants internationaux, trafics de psychotropes et de femmes, blanchiments, Rapport INHES 2009.

THOMAS William I. et ZNANIECKI Florian (1998) Le paysan polonais en Europe et en Amérique. Récit de vie d'un migrant, Paris, Nathan Université, 446 p. 